\title{
High Maneuverability Lenticular Airship
}

\author{
Eduardo Pinto and José Barata \\ Universidade Nova de Lisboa, Faculdade de Ciências e Tecnologia, \\ Departamento de Engenharia Electroténica, \\ 2829-516 Quinta da Torre, Portugal
}

\begin{abstract}
This paper presents and describes a new lenticular shaped airship that allows 3D omnidirectional movements. The airship is intended to be part of a cooperative aerial system based in the synergy between an airship and a group of micro-quad rotors capable of long time missions as environmental, biodiversity monitoring, WiSAR missions, road monitoring, detection of forest fires, etc. The emphasis in this paper is the presentation of hardware of the main component of the system: an innovative lenticular airship, the control algorithm and the low size generic hardware platform specially developed for all the robots in the system..
\end{abstract}

Keywords: Lenticular Airship, WiSAR, Cooperative robots, Lighter than air vehicles.

\section{Introduction}

The actual state of the art of robotics makes very difficult or even impossible to build a single robot with all the capabilities to accomplish very specific missions, namely all that demands long time missions with simultaneous intensive periods of energy consumptions. Taking the example of WiSAR-Wilderness Search and Rescue missions, a robot should be able to cover big distances during long periods of time but also have the ability to access and inspect confined spaces with high maneuverability facing two main requirements:

- Capacity to support variable and unstructured terrain but at the same time low complexity to maintain a high degree of reliability

- High power and communications capacity but at the same time low size to maintain the capacity to deal with a very unstructured environment

These two characteristics are often incompatible since the ability of long periods of operation is directly limited by the size and weight of batteries, what limits maneuverability. We think the best solution is to use two heterogeneous robots working together in an innovative synergetic way. The hypothesis behind our work is how we could use bio-inspired symbiosis relations to improve synergy relations in UAV robots. The solution we propose in this paper is to use two kinds of UAVs working together. The first is a LTAV - Lighter Than Air Vehicle known as a lenticular airship. The airships have inherent characteristics, as: VTOL-vertical 
take-off and landing, natural stability, and hovering capacity with low power consumption. With the use of very thin solar cells based on polyimide film LaRCtrade-CP1 and amorphous silicon it's possible to get a value of energy of $4.3 \mathrm{~W} / \mathrm{g}$ [1] that will allow the airship to be able to do energy harvesting for itself and for the second robot in the system: a micro quadrotor. Recent developments will allow the direct deposit at a low cost of solar cells in almost all kind of materials [2], [3] including the ones used for airships envelopes. We choose the quadrotor configuration since it exhibits high maneuverability even in confined spaces, but with the counterpart of a high power consumption, that limits its operational possibilities. It is our belief that one of the best methods to overpass this difficulty is to implement a synergetic relation between the two robots in the system. During the mission, the robotic system will assess a series of variables defined according to the type of mission, and when we have a specific indicator, the quadrotor platform will be asked to perform a local survey. This information is passed using the long range communication system installed in the airship to a remote control center, where a human operator is monitoring and configuring the mission.

\subsection{Lenticular Airships}

As far we can check, the first lenticular airship design was proposed by Louis Henri Capazza in 15 May 1908 at magazine L'Aérophile: "Le planeur Bayard-Clément". It was a hybrid airship with rudders at the back.This is the same configuration used by Pierre Balaskovic in Alizé project 2008. However, the Bayard-Clément received more interest from the Russian military than the French. General Boreskoff presented the project to the Air Navigation Comission in Saint-Petersburg, but the costs were considered very high. Anyway, the idea had influenced the airship Russian designers until our days (Thermoplan project from Lokomosky) [4]. The difficulty in controlling and building these types of airships, despite its great advantages, has discouraged the designers for adopting this kind of shape, with only some few examples during the XX century [5] as we could see in Fig. 1.

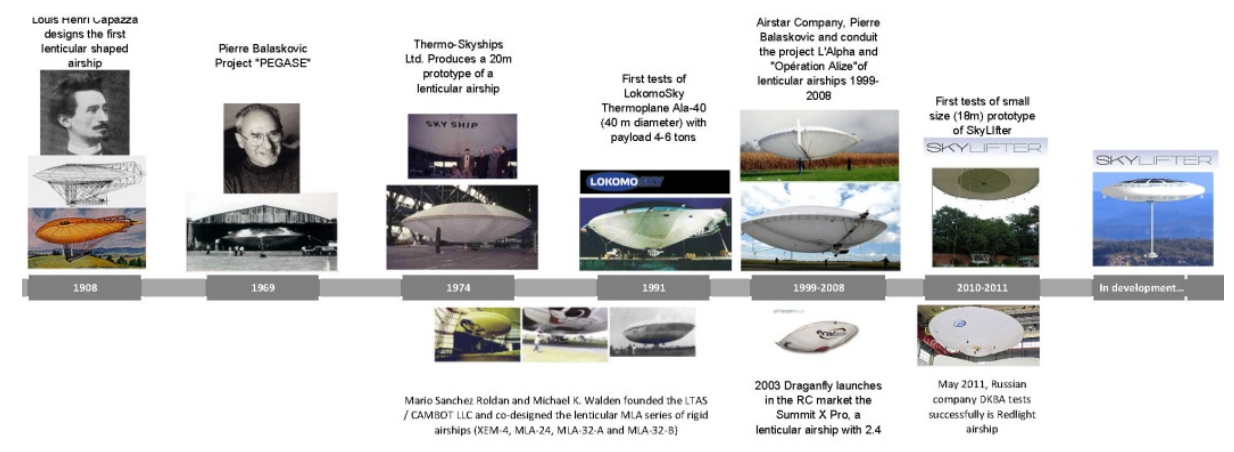

Fig. 1. Lenticular LTAV timeline 


\subsection{Airship Shape Design}

The minimum weight of the airship structure under given buoyant lift can be achieved under sphere-shaped body, but such shape has big drag and that is why classic airship has shape of low drag oblong streamlined body of revolution[6]. However in the last years we had seen airships with very different unconventional shapes. Some examples are pointed in Table 1.

Table 1. Airship common shapes

\begin{tabular}{lcccccc}
\hline Shape & Airship e.g. & Drag & Manover. & Wind & Lift & Moor \\
\hline Cigar-shaped & Hinderburg & ++ & + & + & ++ & - \\
Spherical & Aerosphere SA-60 & - & ++ & + & +++ & + \\
Lenticular & Alizé & ++ & +++ & ++ & + & +++ \\
Multiple Hull & L. Martin P791 & + & + & + & ++ & - \\
Winged airships & BARS "Bella-1" & + & ++ & + & + & - \\
Dart Shape & Bullet Airship & +++ & + & + & ++ & - \\
Deltoid & AEREON 26 & + & ++ & + & + & - \\
\hline
\end{tabular}

The lenticular shape exhibits several advantages for our system. In fact it's the shape that allows the easiest mooring capability without the need for hangars or mooring masts. It's also the shape that presents the greater top surface area, what is very important for the use of solar cells. By presenting the same low drag shape in all directions makes it less sensible to lateral winds and turbulence and allows to implement a true innovative omnidirectional locomotion. The solution used and described in 3.4, allows overcoming the control problems described by other authors, [7],[8] allowing for very stable flight and hovering and vertical land and take off with very simple algorithms.

\section{Contribution to Value Creation}

It's our idea that the work here presented could contribute significantly to value creation by presenting a new kind of airship and a complete control solution (hardware and control algorithm). It's our conviction that the hardware developed, with its small size and weight combining the best MEMS sensors actually available in the market could be used not only as the control board in airships and small UAVs but also for many other applications that require low cost, low size IMUs. As far we could check many lenticular airships projects have been abandoned in the last years because 
of control problems. In the first tests made our control system has proved to be very effective allowing for indoor straight trajectories with minimal errors, and its use could be important to revive the interest for this kind of airships. Also some of public presentations have shown the great appealing of an airship of this kind and the great capacities for publicity use. The high stability and very stable hovering capabilities also opens an enormous range of applications, from environmental, security and as a platform to obtain innovative field of views and high quality images for use in movies and TV shows.

\section{The Lenticular Shaped Airship}

All the materials with the exception of the envelope, manufactured by Mobile Airships according to our drawings, could be easily acquired in Kite and RC specialty stores contributing to maintain the project at a low cost and easily replicable. The main characteristics are pointed in Table 2 .

Table 2. Main characteristics

\begin{tabular}{|c|c|}
\hline Dimensions & $3.2 \mathrm{~m}$ diameter, $80 \mathrm{~cm}$ height (Fully inflated, $5.5 \mathrm{~m}^{3}, \mathrm{He} 98 \%$, ) \\
\hline Propulsion & $\begin{array}{l}4 \text { brushless directional EDF (GWEDF505E \& BL2018-3) with } \\
\text { modified digital servos Graupner HVS930BB }\end{array}$ \\
\hline Outer ring & $11 \mathrm{~mm} x 1 \mathrm{~mm}$ carbon fiber bar with $4 \mathrm{~mm}$ carbon rod \\
\hline Envelope & Custom made, PU 6 mil, $3.5 \mathrm{~m}$ diameter \\
\hline Battery & Kokam LiPo 30C 2S2P 4000mAH \\
\hline Speed & about $4 \mathrm{~m} / \mathrm{s}$ with compensation active ( $2 \mathrm{~m} / \mathrm{s}$ up and down) \\
\hline Payload & $800 \mathrm{~g}$ without sensors \\
\hline Sensors & $\begin{array}{l}\text { IMU with GPS, SRF10 Ultrasonic distance sensors, IR Sharp } \\
\text { distance sensor GP2YDA02 }\end{array}$ \\
\hline Communications & WiFi or Bluetooth $+2.4 \mathrm{GHz}$ Futaba $2.4 \mathrm{GHz}$ FASST module \\
\hline
\end{tabular}

The lenticular shape is maintained by a circular ring made with carbon fiber bar $(11 \mathrm{~mm} \times 1 \mathrm{~m})$ with a $4 \mathrm{~mm}$ carbon rod. This structure is tensioned as in a bicycle wheel with the Kevlar pre-glued wires during the manufacture of the bag. This way we get a very low weight but rigid structure to support the motors with enough flexibility to support and absorb shocks with obstacles. 


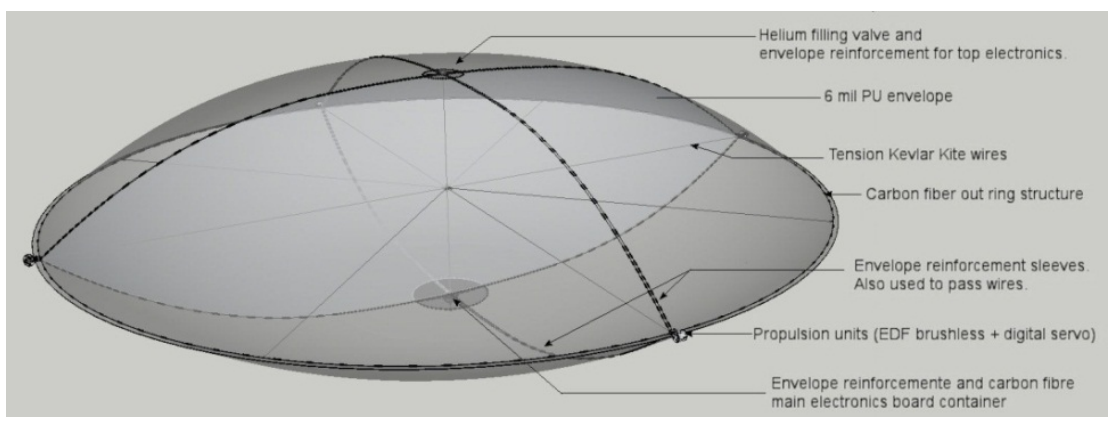

Fig. 2. Airship structure

\subsection{Airship Control Hardware}

The main component of the system is the IMU controller board totally developed by the authors, with the characteristics pointed in Table 3 that are unique in the market, that allows it to be used not only in the airship but in the micro quadrotors.

Table 3. Controller board and IMU characteristics

\begin{tabular}{ll}
\hline Dimensions & $4 \mathrm{~cm} \times$ 4cm, aboutt 20g \\
Microcontroller & MIPS32 M4K 32 bit microcontroller technology with \\
& 1.5DMIPS/MHZ \\
Communication & USB, CAN, I2C, RS485, ICSP\&JTAG\&DEBUG, \& 10 \\
& PIO \\
Wireless & WiFi (MRF24WB0MB) or Bluetooth (RN-41) \\
Sensors & IMU 9 DOF with GPS SiRF starIVTM, Barometer \& \\
& Temperature \\
\hline
\end{tabular}

In Fig. 3, we could observe a block diagram of the airship architecture. The airship uses mainly a CAN channel to interconnect all the main boards. It was also necessary to develop a brushless control board with a CAN interface since we did not find any similar board in the market. It was necessary to modify some commercial servos Graupner HVS930BB to allow a movement of 300 degrees. There were some available servos in the market with 360 or more degrees turn, namely sail wing servos but their reliability and weight did not fit our requirements. In this version we have equipped the airship with 4 IR distance sensors (analog interface, one in each EDF) and 6 SRF10 ultrasonic sensors (I2C interface, one at the top and at the bottom and one in each EDF). 


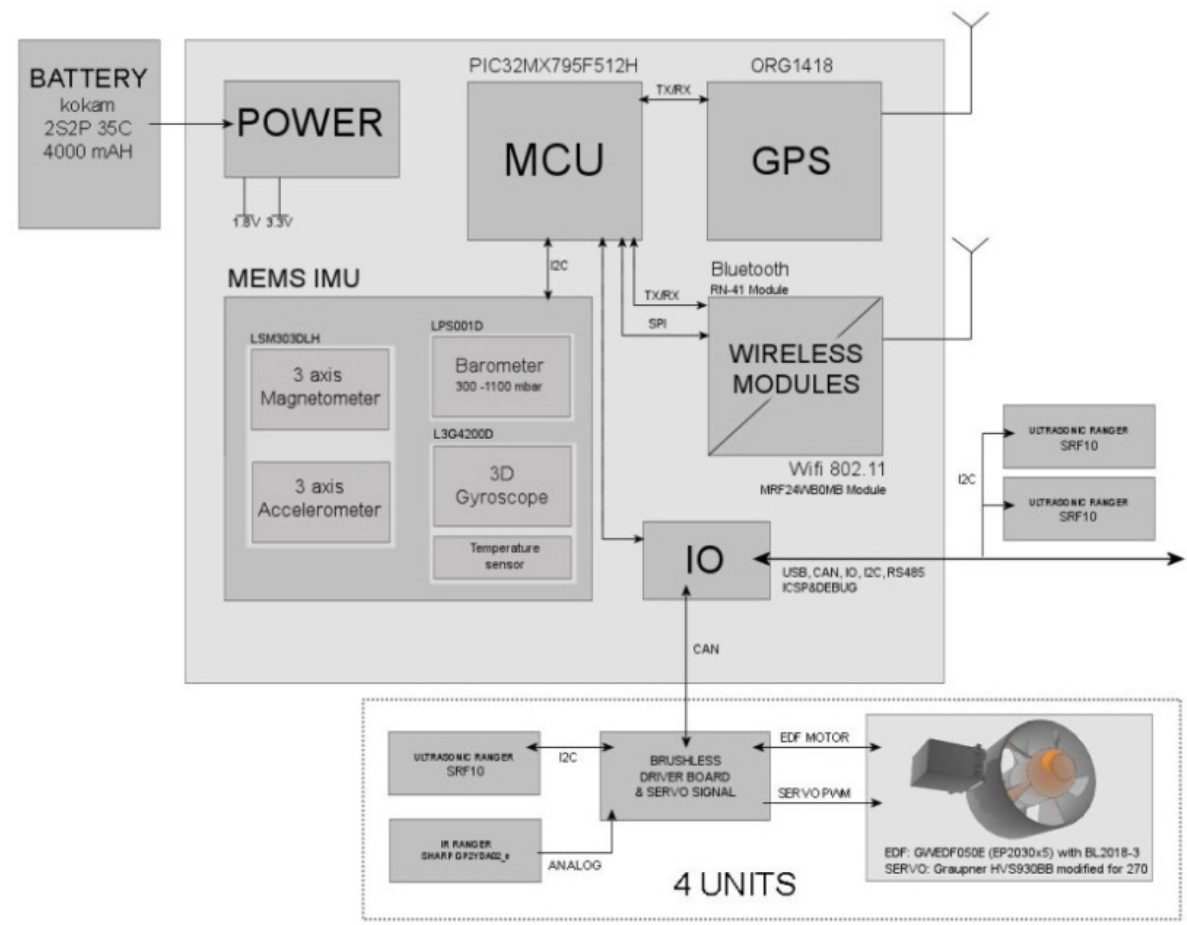

Fig. 3. Block diagram of the airship control hardware
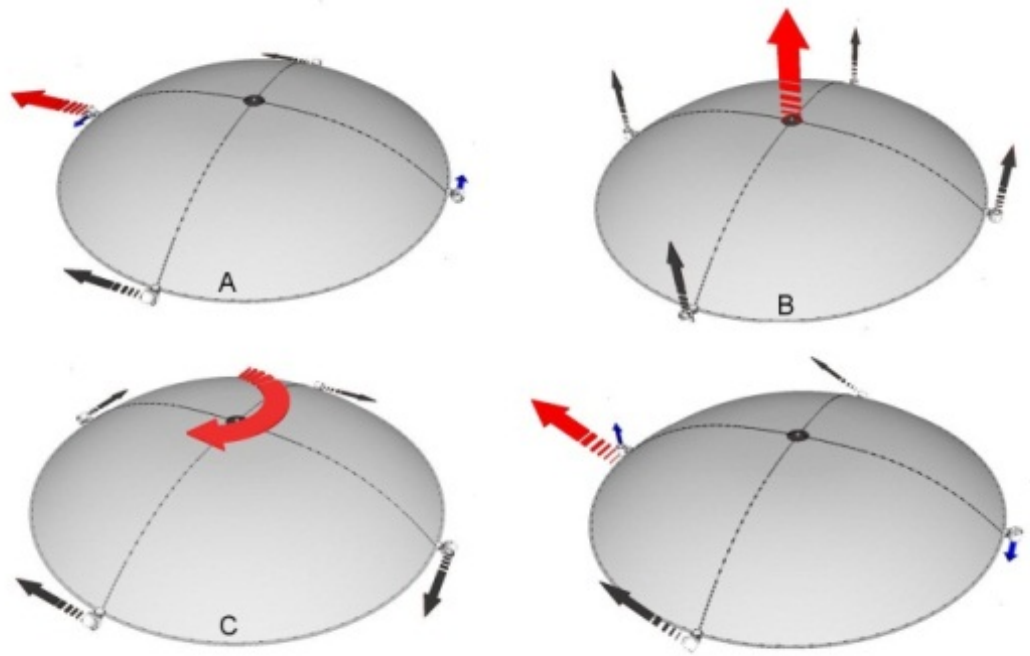

Fig. 4. Locomotion modes 


\subsection{Locomotion Modes}

Basically there are 4 possible locomotion modes as we can see in. All of these modes allow a true omnidirectional locomotion in a 3D space. They are:

A - Straight trajectory in a horizontal plane with automatic compensation of the trajectory. In this mode we use two opposite ducted fans to give the impulse and speed in the desired direction. The other two fans are used for compensation of the trajectory based in the information from the gyro, accelerometer and compass sensors.

Table 4. Angles for each EDF in the different locomotion modes

\begin{tabular}{|c|c|c|c|c|c|}
\hline \multicolumn{6}{|c|}{ MODE "A" - Straight trajectory in a horizontal plane } \\
\hline & EDF 1 & EDF2 & EDF3 & EDF4 & Compensation for \\
\hline Front & $90^{\circ}$ & $90^{\circ}$ & $90^{\circ}$ & $270^{\circ}$ & Straight path \\
\hline Back & $90^{\circ}$ & $270^{\circ}$ & $90^{\circ}$ & $90^{\circ}$ & Straight path \\
\hline Right & $270^{\circ}$ & $90^{\circ}$ & $90^{\circ}$ & $90^{\circ}$ & Straight path \\
\hline Left & $90^{\circ}$ & $90^{\circ}$ & $270^{\circ}$ & $90^{\circ}$ & Straight path \\
\hline \multicolumn{6}{|c|}{ MODE “B” - Vertical trajectory } \\
\hline $\mathrm{Up}$ & $0^{\circ}$ & $0^{\circ}$ & $\boldsymbol{0}^{\circ}$ & $\boldsymbol{0}^{\circ}$ & Horizontality \\
\hline Down & $180^{\circ}$ & $180^{\circ}$ & $180^{\circ}$ & $180^{\circ}$ & Horizontality \\
\hline \multicolumn{6}{|c|}{ MODE “C” - Rotation } \\
\hline $\mathrm{CW}$ & $270^{\circ}$ & $270^{\circ}$ & $270^{\circ}$ & $270^{\circ}$ & Rotational speed \\
\hline $\mathrm{CCW}$ & $90^{\circ}$ & $90^{\circ}$ & $90^{\circ}$ & $90^{\circ}$ & Rotational speed \\
\hline \multicolumn{6}{|c|}{ MODE "D" - Trajectory in a inclined plane } \\
\hline Front & $0^{\circ}$ & $90^{\circ}-\alpha$ & $180^{\circ}$ & $270^{\circ}+\alpha$ & Inclination $(\boldsymbol{\alpha})$ \\
\hline Back & $180^{\circ}$ & $270^{\circ}+\alpha$ & $0^{\circ}$ & $90^{\circ}-\alpha$ & Inclination \\
\hline Right & $270^{\circ}+\alpha$ & $0^{\circ}$ & $90^{\circ}-\alpha$ & $180^{\circ}$ & Inclination \\
\hline Left & $90^{\circ}-\alpha$ & $180^{\circ}$ & $270^{\circ}+\alpha$ & $0^{\circ}$ & Inclination \\
\hline
\end{tabular}

Notes: Italic: Ducted fan used for compensation.

Bold: Ducted fan used for trust.

Angles and numeration according to the following diagram:

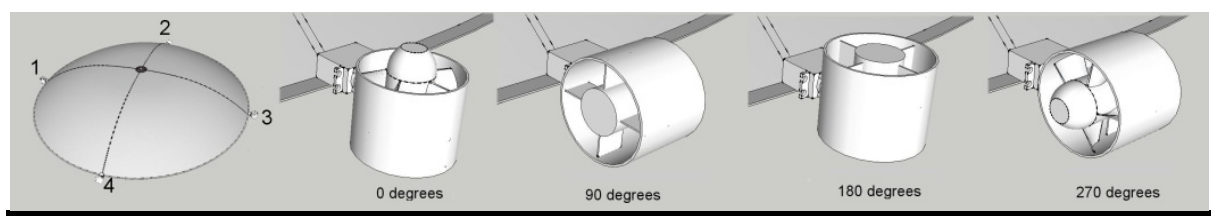


B - This locomotion mode allows vertical takeoff and landing. All of the turbines are pointed to the trajectory to follow. The attitude and speed of the airship is controlled, by changing individually the speed of each turbine. In this mode the drag imposed by the great and orthogonal surface is easily overcome by the simultaneous trust of the 4 turbines.

C - Rotation. This mode allows very quick changes in direction. It is possible to make very fast rotations that allows for example to get a very rapid 360 degrees sequence of photos.

D - In this mode the airship could climb or descent in an inclined plan. As in mode "A" two of the ducted fans are used for giving the trust and the other two to maintain the trajectory in the inclined plane. The maximum inclination is $30^{\circ}$, limited by the angular range of the servos. Experiences made with inclinations near this limit show that it is very difficult to maintain stability and follow a straight path. The compensation is mandatory since the shape of the airship maintains by itself the airship stable in a horizontal plan.

The Table 4, resumes all the locomotion modes and how the control algorithm implements the angle for each propulsion unit and if the EDF is used for propulsion or for compensation of the movement.

Since there is more than one possible solution, special care has been taken to get the best solution possible in terms of energetic consumption and to limit the number of servo movements between modes transitions.

The control algorithm used is very simple and uses a PID controller to obtain the desired movement based on the information filtered by a very simple digital median filter of the sensors implied in each mode. By example, when we pretend to follow a straight path forward we use the gyroscope data as input for a PID controller integrated in a feedback loop to try to minimize the difference between the desired direction and the actual one.

\section{Conclusions and Further Work}

At the current time we are doing some experiences and trying variations on the control algorithm, but the first flight tests done in controlled indoor conditions with the simple control algorithm presented in this paper, were very encouraging. Indeed the hover capability and the ability to stay stationary at a given altitude exceeded all our expectations in the testes made, with the airship perfectly stationary at different altitudes. Also the maneuver capacities were excellent and allowed us to verify the great advantages of a lenticular shape when compared with the traditional blimp shape. Our airship could easily do movements as instantaneous direction changes, rotation in a fixed point and climb up and down in a vertical trajectory. For testing the autonomous capabilities of the airship we conducted two tests. The first one was to follow a straight rectangular trajectory, parallel to walls using omnidirectional 
changes of direction at the corners, using only the ultrasonic sensors to maintain a fixed distance to walls. The airship has done this flight with very success maintaining always a straight trajectory, with some derivation at the 90 degrees angles we imposed at corners.
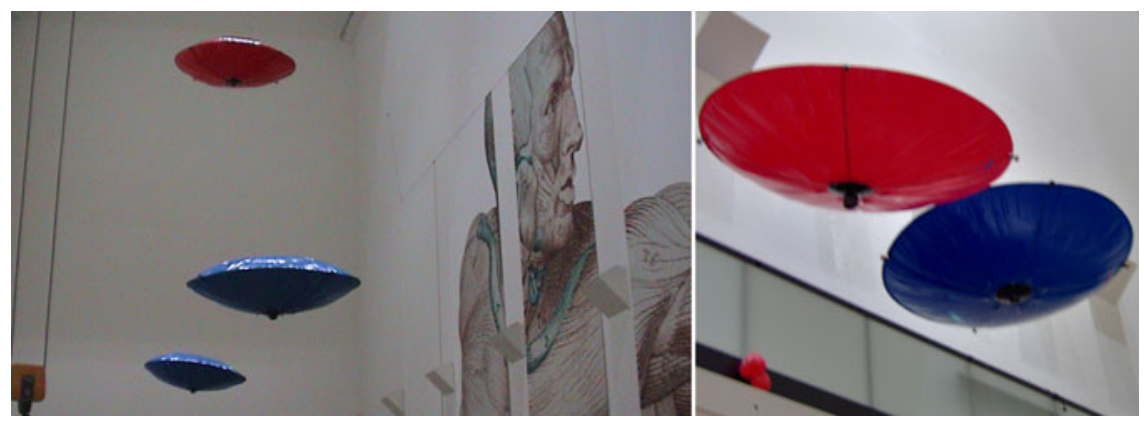

Fig. 5. Airships during tests at "Pavilhão do Conhecimento"

In the second experience we used the data from the ultrasonic sensors to maintain a formation with 3 airships in a controlled indoor situation, and to avoid obstacles. The experience had revealed very good results with the airships in a plane and the use of sensors to avoid obstacles as walls, but also revealed that is necessary to use other type of solution to maintain a 3D formation and avoid collisions as the one we can see in Fig. 5. At the moment we are trying some low cost color based image recognition modules and IR cameras as the Wii camera.

Acknowledgments. The authors would like to thank the governmental science agency "Ciência Viva" for the financial contribute to the first prototypes and for allowing us to make some experiences in pavilion of Knowledge in "Parque das Nações", Lisbon.

\section{References}

1. Wyrsch, N., et al.: Ultra-Light Amorphous Silicon Cell for Space Applications. In: Proc. of IEEE 4th World Conference on Photovoltaic Energy Conversion, Waikoloa (2006)

2. Rowehl, J.A., Lunt, R.R., Xu, J., Wang, A., Boyce, C.M., Gap Im, C., Bulovic, V., Gleason Mile, K.K.: Direct Monolithic Integration of Organic Photovoltaic Circuits on Unmodified Paper, Advanced Materials (2011)

3. Airshipworld (2009), http: / / airshipworld.blogspot.com/

4. Alexander Filimonov. Tyumenekotrans (2002), http: / /www . tumenecotrans . ru

5. Liao, L., Pasternak, I.: A review of airship structural research and development. Progress in Aerospace Sciences 45 (2009) 
6. Konstantinov, L.: The Basics of Gas and Heat Airship Theory (2005),

http: / /www .agaeroplast. com

7. Balaskovic, P.,

http: //balaskovic . pagesperso-orange. fr/ballons-et-

dirigeables.html

8. Bonora, N.: Nuove prospective per l'Areonave, CeMISS - Centro Militare di Studi Strategici. Rubbentino Editore, Roma (2005) 